03

\title{
Влияние продольного электрического поля на капиллярную неустойчивость тонкого осесимметричного слоя жидкого диэлектрика, покрывающего диэлектрическую нить
}

\author{
(C) В.М. Коровин \\ Московский государственный университет им. М.В. Ломоносова, НИИ механики МГУ, \\ 119192 Москва, Россия \\ e-mail: verazhan@yandex.ru
}

(Поступило в Редакцию 10 октября 2014 г. В окончательной редакции 14 апреля 2016 г.)

\begin{abstract}
Исследовано движение вязкой диэлектрической жидкости, окруженной газом, в процессе капиллярного распада тонкого осесимметричного жидкого слоя на недеформируемой диэлектрической нити цилиндрической формы, находящейся в однородном продольном электрическом поле. Применительно к случаю, когда средняя толщина слоя много меньше радиуса поперечного сечения нити, проведен асимптотический анализ системы уравнений и граничных условий гидродинамики, записанных с учетом поверхностных пондеромоторных сил. Сформулирована постановка задачи о переходе жидкой конфигурации из состояния покоящегося цилиндрического слоя в гидростатическое состояние в виде регулярной последовательности капелек. В рамках предложенной постановки выведено нелинейное параболическое уравнение, описывающее в длинноволновом приближении эволюцию локальной толщины слоя на интервале времени до момента образования капелек. На базе линеаризованной версии полученного уравнения и линеаризованной задачи электростатики о расчете возмущений поля исследовано влияние определяющих параметров на капиллярную неустойчивость.
\end{abstract}

\section{Введение}

В результате проведенных во второй половине ХІХ в. экспериментов со слоем вязкой жидкости (касторовое масло), нанесенной на кварцевую нить, стало известно [1], что сплошной слой, покрывающий нить, быстро распадается на шарообразные капельки, подобно бусинкам, „нанизанным“ на нить. Образующиеся капельки располагаются периодическим образом вдоль нити на значительном расстоянии друг от друга. Наряду с крупными капельками (основной размер) за счет нелинейных эффектов формируются сателлиты - более мелкие капельки, лежащие в середине каждого промежутка между капельками основного размера. Наблюдается также дальнейшее продолжение этого процесса - на нити между капелькой основного размера и сателлитом появляется еще более мелкая капелька.

Причиной перехода первоначально сплошного цилиндрического слоя в гидростатическое состояние в виде регулярной последовательности капелек, как и в случае неустойчивости жидкого цилиндра $[2,3]$, является неустойчивость Плато-Рэлея, вызываемая капиллярными силами, стремящимися минимизировать площадь свободной поверхности. Одним из примеров такой перестройки и формирования подобной жидкой конфигурации является природный объект - нить паутины [1].

Аналогичная картина распада на капли жидкого слоя, образующегося на стеклянной нити радиуса $0.016 \mathrm{~mm}$ при ее извлечении из трансформаторного масла, наблюдалась в работе [4]. В эксперименте отношение толщины слоя к радиусу нити составляло 0.72. Независимость толщины слоя как от расстояния вдоль извлеченной из жидкости части нити, так и от угла наклона нити к горизонтальной поверхности жидкости, из которой она извлекается, свидетельствуют, что влияние силы тяжести на формирование слоя и его распад несущественно [4,5].

Знание особенностей развития капиллярной неустойчивости жидкого слоя на твердом цилиндре малого радиуса требуется для оптимизации ряда технологических процессов, в частности, при нанесении покрытий на проволочки и синтетические волокна [5].

В стандартной постановке теоретическое исследование капиллярной неустойчивости изотермического жидкого слоя, покрывающего проволочку, базируется на использовании уравнений и граничных условий гидродинамики вязкой жидкости, линеаризованных относительно гидростатического состояния жидкости с цилиндрической свободной поверхностью [6].

В отличие от [6] в работе [7] при выводе квазиодномерных уравнений, описывающих движение вязкой неравномерно нагретой жидкости в осесимметричном слое со свободной поверхностью, покрывающем цилиндр малого радиуса, используются уравнения баланса массы, импульса и тепловой энергии в выделенном объеме между двумя близкими поперечными сечениями слоя.

В обзоре [8] изложена имеющая множество приложений процедура построения на базе полных уравнений Навье-Стокса более простой нелинейной модели длинноволнового движения жидкости в тонком плоском слое со свободной поверхностью. При выводе основополагающего в этом подходе эволюционного уравнения привлекаются допущения, реализуемые при гидродинамической смазке [9]. 
Известно, что однородное электрическое поле, параллельное свободной поверхности жидкого диэлектрика, оказывает стабилизирующее воздействие на вызываемые различными факторами неустойчивости поверхностей раздела диэлектрическая жидкость-газ: на капиллярную неустойчивость жидкого цилиндра [10], на сверхтонкий (толщиной 100-1000 А) плоский слой диэлектрической жидкости, динамика которой в значительной степени определяется расклинивающим давлением, создаваемым силами Ван-дер-Ваальса [11], на неустойчивость Рэлея-Тейлора [12].

В настоящей работе изучен капиллярный распад осесимметричного слоя, не содержащего свободных электрических зарядов жидкого диэлектрика с диэлектрической проницаемостью $\varepsilon_{1}$, покрывающего находящуюся в однородном продольном электрическом поле $\mathbf{E}_{0}$ недеформируемую прямолинейную цилиндрическую нить. Проницаемость материала нити $\varepsilon_{2} \geq \varepsilon_{1}$. Предполагается, что средняя толщина слоя мала по сравнению с радиусом поперечного сечения нити. Вывод эволюционного уравнения проведен с учетом поверхностных пондеромоторных сил, создаваемых приложенным полем.

Целью работы является исследование влияния определяющих параметров на характеристики капиллярной неустойчивости: пространственный масштаб и скорость роста наиболее быстро растущей моды, нейтральную кривую, область неустойчивости.

\section{Определяющие уравнения}

Рассматривается вызываемое силами поверхностного натяжения движение вязкой диэлектрической жидкости с проницаемостью $\varepsilon_{1}$ при капиллярном распаде цилиндрического в начальный момент времени неподвижного слоя, покрывающего диэлектрическую нить с проницаемостью $\varepsilon_{2} \geq \varepsilon_{1}$. Нить и жидкость находятся в однородном продольном электрическом поле $\mathbf{E}_{0}$, созданном внешним устройством (рис. 1). Снаружи жидкий слой окружен газом, давление в котором $p_{a}$ постоянно. Предполагается, что $E_{0}$ не превышает пробивное значение, составляющее для сухого воздуха $30 \mathrm{kV} / \mathrm{cm}$ [13]. Далее, как и в [13], используется гауссова система CGS.

Система уравнений и граничных условий электрогидродинамики в рамках модели Тейлора-Мелчера [14] является итогом многочисленных работ, выполненных во второй половине $\mathrm{XX}$ в. Эта модель учитывает утечку из рассматриваемого объема жидкого диэлектрика свободных электрических зарядов как поступающих в объем извне, так и образующихся за счет различных физико-химических процессов в самом объеме и на его границе.

В электрогидродинамике используется также приближение однородного по температуре и другим физическим характеристикам идеального несжимаемого жидкого диэлектрика, не содержащего свободных электрических зарядов (см., например, [10,11,15-17]). Осно-

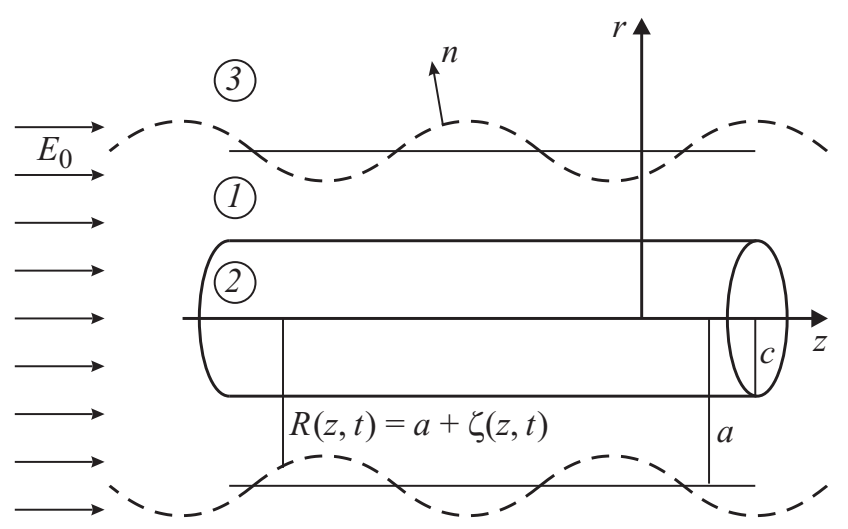

Pис. 1. Геометрия задачи и обозначения.

вы соответствующей теории были заложены в конце XIX в. [13]. При этом математическая запись пондеромоторных сил является частным случаем общих формул электродинамики сплошных сред [18]. Ввиду отсутствия свободных электрических зарядов объемные пондеромоторные силы отсутствуют, так что в рамках такой модели электрическое поле оказывает силовое воздействие на жидкость лишь за счет пондеромоторных сил, локализованных на поверхности раздела с газом или с жидкостью, имеющей другую диэлектрическую проницаемость $[13,18]$.

Обозначим через $\rho, \eta, \alpha, g$ соответственно плотность жидкости, ее динамическую вязкость, коэффициент поверхностного натяжения и ускорение силы тяжести. Пусть $c$ - радиус поперечного сечения нити, а $\delta$ средняя толщина слоя.

Помимо $\varepsilon_{1}, \varepsilon_{2}$ и геометрического фактора $\delta / c$ безразмерными параметрами подобия, характеризующими исследуемое явление, являются число Бонда $B o=$ $=(c+\delta)^{2} / l_{c}^{2}$, число Онезорге $O h=\eta^{2} /[\rho \alpha(c+\delta)]$ и электрокапиллярное число $N=(c+\delta) E_{0}^{2} / \alpha$. Здесь $l_{c}=\sqrt{\alpha /(\rho g)}-$ капиллярная длина.

Предполагается, что в рассматриваемом случае $B o \ll 1$. При выполнении этого условия влияние силы тяжести на форму свободной поверхности жидкости пренебрежимо мало [5] и, как показывают проведенные в отсутствие поля эксперименты [6], движение жидкости осесимметрично и не имеет закрутки. В этой связи следует отметить, что в задаче Рэлея цилиндрическая струя является устойчивой по отношению ко всем возмущениям, которые не являются осесимметричными [3].

Введем цилиндрическую систему координат $r, \vartheta, z$, ось $z$ которой является осью нити, и обозначим $\mathbf{a}_{r}, \mathbf{a}_{z}$ единичные векторы вдоль соответствующих координатных линий. Пусть уравнение $r=R(z, t)$, где $t-$ время, представляет форму свободной поверхности слоя.

Движение жидкости описывается уравнением неразрывности и уравнениями Навье-Стокса

$$
\frac{\partial u}{\partial r}+\frac{u}{r}+\frac{\partial w}{\partial z}=0
$$




$$
\begin{gathered}
\rho\left(\frac{\partial u}{\partial t}+u \frac{\partial u}{\partial r}+w \frac{\partial u}{\partial z}\right)=-\frac{\partial p}{\partial r}+\eta\left(\Delta u-\frac{u}{r^{2}}\right) \\
\rho\left(\frac{\partial w}{\partial t}+u \frac{\partial w}{\partial r}+w \frac{\partial w}{\partial z}\right)=-\frac{\partial p}{\partial r}+\eta \Delta w \\
\Delta=\frac{\partial^{2}}{\partial r^{2}}+\frac{1}{r} \frac{\partial}{\partial r}+\frac{\partial^{2}}{\partial z^{2}} .
\end{gathered}
$$

Здесь $u(r, z, t), w(r, z, t)$ - проекции вектора скорости на координатные линии $r$ и $z$, а $p(r, z, t)$ - давление жидкости.

Будем отмечать индексами $j=1,2,3$ электрическое поле $E_{j}(r, z, t)=-\operatorname{grad} \Phi_{j}$ в слое $(j=1)$, в нити $(j=2)$ и в окружающем газе $(j=3)$. Ввиду отсутствия в жидкости свободных электрических зарядов распределения потенциалов $\Phi_{j}(r, z, t)$ всюду описываются уравнением Лапласа

$$
\Delta \Phi_{j}=0, \quad j=1,2,3 .
$$

Граничные условия электростатики на поверхностях раздела сред с различными диэлектрическими свойствами выражают непрерывность тангенциальной компоненты электрического поля и непрерывность нормальной компоненты вектора индукции:

при $r=c$ :

$$
\Phi_{1}=\Phi_{2}, \quad \varepsilon_{1} \frac{\partial \Phi_{1}}{\partial r}=\varepsilon_{2} \frac{\partial \Phi_{2}}{\partial r}
$$

при $r=R(z, t)$ :

$$
\Phi_{1}=\Phi_{3}, \quad \varepsilon_{1} \frac{\partial \Phi_{1}}{\partial n}=\frac{\partial \Phi_{3}}{\partial n} .
$$

Здесь $\mathbf{n}=\left(n_{r}, 0, n_{z}\right)$ - единичный вектор внешней к свободной поверхности нормали

$$
\mathbf{n}(z, t)=\frac{1}{\sqrt{1+\left(\frac{\partial R}{\partial z}\right)^{2}}}\left(\mathbf{a}_{r}-\frac{\partial R}{\partial z} \mathbf{a}_{z}\right) .
$$

Вдали от жидкого слоя поле однородно и направлено вдоль оси $z$ : при $\frac{r}{c} \rightarrow \infty$ :

$$
\frac{\partial \Phi_{3}}{\partial z}=-E_{0}
$$

На свободной поверхности $r=R(z, t)$ должны выполняться кинематическое условие

$$
\frac{\partial R}{\partial t}+w \frac{\partial R}{\partial z}=u
$$

и динамические условия

$$
\begin{gathered}
p-p_{a}=\alpha \operatorname{divn}+\frac{2 \eta}{1+\left(\frac{\partial R}{\partial z}\right)^{2}}\left[\frac{\partial u}{\partial r}-\frac{\partial R}{\partial z}\left(\frac{\partial w}{\partial r}+\frac{\partial u}{\partial z}\right)\right. \\
\left.+\left(\frac{\partial R}{\partial z}\right)^{2} \frac{\partial w}{\partial z}\right]-\frac{\varepsilon_{1}-1}{8 \pi\left[1+\left(\frac{\partial R}{\partial z}\right)^{2}\right]} \\
\quad \times\left[\varepsilon_{1}\left(\frac{\partial \Phi_{1}}{\partial r}-\frac{\partial \Phi_{1}}{\partial z} \frac{\partial R}{\partial z}\right)^{2}+\left(\frac{\partial \Phi_{1}}{\partial r} \frac{\partial R}{\partial z}+\frac{\partial \Phi_{1}}{\partial z}\right)^{2}\right],
\end{gathered}
$$

$$
\left[1-\left(\frac{\partial R}{\partial z}\right)^{2}\right]\left(\frac{\partial w}{\partial r}+\frac{\partial u}{\partial z}\right)+\frac{\partial R}{\partial z}\left(\frac{\partial u}{\partial z}-\frac{\partial w}{\partial z}\right)=0 .
$$

Кинематическое условие (8) выражает равенство скоростей удаления точек свободной поверхности от оси симметрии и радиальных скоростей жидких частиц, образующих свободную поверхность. Условие (9) выражает равенство нормальных напряжений на противоположных сторонах свободной поверхности. Пропорциональное $\varepsilon_{1}-1$ слагаемое описывает вклад поверхностных пондеромоторных сил [18]. Условие (10) выражает отсутствие касательных напряжений на внешней границе слоя.

На поверхности нити выполняются условия при $r=c$ :

$$
u=0, \quad w=0 .
$$

Задача (1)-(11) имеет точное решение

$$
\begin{gathered}
u=0, w=0, R=c+\delta, p_{0}=p_{a}+\frac{\alpha}{a}-\frac{\varepsilon_{1}-1}{8 \pi} E_{0}^{2}, \\
a=c+\delta, \Phi_{j}^{0}=-E_{0} z, j=1,2,3,
\end{gathered}
$$

описывающее гидростатическое состояние жидкости, образующей слой постоянной толщины $\delta$, поляризованный однородным продольным полем, вызывающим уменьшение давления, создаваемого капиллярными силами.

Сделаем линейную замену части искомых функций

$$
\begin{array}{r}
R(z, t)=a+\zeta(z, t), \quad p(r, z, t)=p_{0}+p_{1}(r, z, t), \\
\Phi_{j}(r, z, t)=\Phi_{j}^{0}(z)+\varphi_{j}(r, z, t), \quad j=1,2,3 .
\end{array}
$$

Отметим, что в рамках нелинейной постановки задачи $\zeta(z, t) \sim \delta$. При записи потенциала в виде $(12)$ уравнения (4) и граничные условия $(5),(6)$, естественно, не изменяются, но задача электростатики формулируется для функций $\varphi_{j}(r, z, t), j=1,2,3$, а на бесконечности (7) ставится условие $\partial \varphi_{3} / \partial z \rightarrow 0$ при $r / c \rightarrow \infty$.

\section{Постановка задачи}

Исследуем влияние пондеромоторных сил на развитие капиллярной неустойчивости осесимметричного слоя, характерная толщина которого много меньше радиуса поперечного сечения нити: $\delta / c \ll 1$. Покажем, что в этом случае после отбрасывания малых величин в определяющих уравнениях и граничных условиях (1)-(11), преобразованных с учетом (12), задача существенно упрощается.

Обратимся к численным данным, полученным в отсутствие поля при экспериментальном исследовании капиллярного распада слоя касторового масла на тонкой проволочке [19]. Обозначим через $\kappa_{m}=k_{m}(c+\delta)$ безразмерное волновое число наиболее быстро растущей моды. Согласно [19], для характеризовавших эксперимент пар величин $c, \delta$, последовательно имевших значения: $0.0762, \quad 0.1619 \mathrm{~mm} ; 0.125,0.125 \mathrm{~mm} ; 0.254,0.015 \mathrm{~mm}$ 
были зарегистрированы соответственно следующие безразмерные волновые числа: $\kappa_{m}=0.68,0.67,0.66$. Эти результаты показывают, что величина отношения $\delta / c$ слабо влияет на $\kappa_{m}$. Полученные значения не слишком сильно отличаются от теоретической величины $\kappa_{m}=1 / \sqrt{2}$, вычисленной в задаче об устойчивости цилиндрической струи радиуса $a=c+\delta$ [3]. В этой задаче все моды с волновыми числами $k$, для которых $k a<1$, неустойчивы, тогда как мода с $k_{0}=a^{-1}$ нейтрально устойчива, а моды с $k a>1$ устойчивы.

C учетом вышесказанного в качестве характерного пространственного масштаба мод в задаче о капиллярной неустойчивости диэлектрического слоя выберем величину $\lambda_{0}=2 \pi k_{0}^{-1}$. Введем безразмерный параметр $\gamma=2 \pi \delta / \lambda_{0}=\delta / a$. В качестве численного примера укажем, что в случае, когда $c=0.25 \mathrm{~mm}$, имеем $\gamma=0.074$ при $\delta=0.02 \mathrm{~mm} ; \gamma=0.17$ при $\delta=0.05 \mathrm{~mm}$.

Предполагая выполненным условие $\gamma \ll 1$ (что эквивалентно $\delta / c \ll 1)$, запишем соответствующее этому случаю приближение (называемое длинноволновым) рассматриваемой задачи.

В длинноволновом приближении лидирующий член разложения по $\gamma$ выражения единичного вектора внешней к свободной поверхности нормали имеет вид $\mathbf{n}(z, t)=\mathbf{a}_{r}-\frac{\partial \xi}{\partial z} \mathbf{a}_{z}$. С учетом этой формулы второе граничное условие (6) записывается следующим образом: при $r=a+\zeta(z, t)$ :

$$
\varepsilon_{1} \frac{\partial \varphi_{1}}{\partial r}-\frac{\partial \varphi_{3}}{\partial r}=\frac{\partial \xi}{\partial z}\left[\left(1-\varepsilon_{1}\right) E_{0}-\frac{\partial \varphi_{3}}{\partial z}+\varepsilon_{1} \frac{\partial \varphi_{1}}{\partial z}\right] .
$$

Далее, подставив (12) в граничные условия (8)-(10) на свободной поверхности $r=a+\zeta(z, t)$ при учете лишь лидирующего члена разложения $\mathbf{n}(r, z, t)$, имеем

$$
\begin{gathered}
\frac{\partial \xi}{\partial t}+w \frac{\xi}{\partial z}=u \\
p_{1}=-\frac{\alpha}{a^{2}}\left(\xi+a^{2} \frac{\partial^{2} \xi}{\partial z^{2}}\right)+2 \eta\left[\frac{\partial u}{\partial r}-\frac{\partial \xi}{\partial z}\left(\frac{\partial w}{\partial r}+\frac{\partial u}{\partial z}\right)\right. \\
\left.+\left(\frac{\partial \xi}{\partial z}\right)^{2} \frac{\partial w}{\partial z}\right]-\Pi_{e}
\end{gathered}
$$

где

$$
\begin{aligned}
& \Pi_{e}= \frac{\varepsilon_{1}-1}{8 \pi}\left\{\varepsilon_{1}\left[\frac{\partial \varphi_{1}}{\partial r}+\frac{\partial \xi}{\partial z}\left(E_{0}-\frac{\partial \varphi_{1}}{\partial z}\right)\right]^{2}\right. \\
&+\left.\left(\frac{\partial \varphi_{1}}{\partial z}+\frac{\partial \xi}{\partial z} \frac{\partial \varphi_{1}}{\partial r}\right)^{2}-2 E_{0}\left(\frac{\partial \varphi_{1}}{\partial z}+\frac{\partial \xi}{\partial z} \frac{\partial \varphi_{1}}{\partial r}\right)\right\} \\
& \frac{\partial w}{\partial r}+\frac{\partial u}{\partial z}+\frac{\partial \xi}{\partial z}\left(\frac{\partial u}{\partial r}-\frac{\partial w}{\partial z}\right)=0
\end{aligned}
$$

В общем случае все слагаемые в (13) по порядку величины одинаковы, ввиду чего на свободной поверхности при $\varepsilon_{1}-1 \sim \varepsilon_{1}$ имеем

$$
\frac{\partial \varphi_{1}}{\partial r} \sim E_{0} \frac{\partial \xi}{\partial z}, \quad \frac{\partial \varphi_{1}}{\partial z} \sim E_{0}
$$

С учетом этих оценок с точностью до малых высшего порядка получаем

$$
\Pi_{e}=\left.\frac{\varepsilon_{1}-1}{8 \pi} \frac{\partial \varphi_{1}}{\partial z}\left(\frac{\partial \varphi_{1}}{\partial z}-2 E_{0}\right)\right|_{r=a+\xi(z, t)} .
$$

При проведении оценок в уравнениях (1)-(3) и в граничных условиях гидродинамики (14)-(16) с целью упрощения введем переменную $x=r-c$, область изменения которой $0 \leq x \leq \delta+\zeta(z, t)$ совпадает с протяженностью в радиальном направлении области, занятой жидкостью.

Конкретизируем рассматриваемое движение жидкости. С этой целью укажем характерные величины определяющих параметров.

Поскольку движение вызывается капиллярными силами (по крайней мере в отсутствие электрического поля), то из динамического условия (15) следует, что характерное отклонение давления в слое $p_{1}^{0}$ от гидростатического давления по порядку величины равно $p_{1}^{0} \sim \delta \alpha / a^{2} \sim \gamma \alpha / a$.

Далее рассматривается режим течения для которого, как и в гидродинамической теории смазки [9], имеется баланс сил трения и продольного градиента давления: $\partial p_{1} / \partial z \sim \eta \partial^{2} w / \partial x^{2}$. Из этого условия легко оценить характерную величину продольной скорости: $w_{0} \sim p_{1}^{0} \delta^{2} /\left(\eta \lambda_{0}\right) \sim \gamma^{3} \alpha / \eta$.

Из уравнения неразрывности (1) следует оценка $u_{0} \sim \gamma^{4} \alpha / \eta$. При этом характерное время имеет порядок $\delta / u_{0} \sim \delta \eta /\left(\gamma^{4} \alpha\right)$.

Отметим, что в постановках задач механики сплошной среды число определяющих параметров обычно больше числа основных единиц измерения используемой системы (см., например, [20]).

Используя выписанные характерные величины, введем безразмерные переменные и искомые функции (со звездочками)

$$
\begin{gathered}
x=\delta x_{*}, z=\frac{\delta}{\gamma} z_{*}, t=\frac{\delta \eta}{\gamma^{4} \alpha} t_{*}, \xi=\delta \xi_{*}\left(z_{*}, t_{*}\right), \\
u=\frac{\gamma^{4} \alpha}{\eta} u_{*}\left(x_{*}, z_{*}, t_{*}\right), w=\frac{\gamma^{3} \alpha}{\eta} w_{*}\left(x_{*}, z_{*}, t_{*}\right), \\
p_{1}=\frac{\gamma^{2} \alpha}{\delta} p_{1}^{*}\left(x_{*}, z_{*}, t_{*}\right), \varphi_{1}=E_{0} a \varphi_{1}^{*}\left(r, z_{*}, t_{*}\right) .
\end{gathered}
$$

В правые части этих равенств безразмерный малый параметр $\gamma$ входит в разных степенях, поскольку в задаче имеется 2 характерных пространственных масштаба, а характерные значения параметров, определяющих рассматриваемый режим осесимметричного течения, получены путем оценок на базе системы дифференциальных уравнений в частных производных и граничных условий, содержащих производные по координатам разных порядков.

После перехода в уравнениях (1)-(3) к безразмерным переменным получаем (звездочки опущены)

$$
\frac{\partial u}{\partial x}+\frac{\gamma u}{\gamma x+1-\gamma}+\frac{\partial w}{\partial z}=0
$$




$$
\begin{aligned}
& \frac{\gamma^{7}}{O h}\left(\frac{\partial u}{\partial t}+u \frac{\partial u}{\partial x}+w \frac{\partial u}{\partial z}\right)=-\frac{\partial p_{1}}{\partial x}+\gamma^{2}\left[\frac{\partial^{2} u}{\partial x^{2}}\right. \\
& \left.+\frac{\gamma}{\gamma x+1-\gamma} \frac{\partial u}{\partial x}+\gamma^{2} \frac{\partial^{2} u}{\partial z^{2}}-\frac{\gamma^{2} u}{(\gamma x+1-\gamma)^{2}}\right], \\
& \frac{\gamma^{5}}{O h}\left(\frac{\partial w}{\partial t}+u \frac{\partial w}{\partial x}+w \frac{\partial w}{\partial z}\right)=-\frac{\partial p_{1}}{\partial z}+\frac{\partial^{2} w}{\partial z^{2}} \\
& +\frac{\gamma}{\gamma x+1-\gamma} \frac{\partial w}{\partial x}+\gamma^{2} \frac{\partial^{2} w}{\partial z^{2}}-\frac{\gamma^{2} w}{(\gamma x+1-\gamma)^{2}},
\end{aligned}
$$

где $O h$ - ранее введенное число Онезорге. В безразмерных переменных кинематическое условие на свободной поверхности сохраняет форму записи (14), а динамические условия (15), (16) с учетом (17) принимают вид

$$
\begin{gathered}
p_{1}=-\left(\xi+\frac{\partial^{2} \xi}{\partial z^{2}}\right)+2\left[\gamma^{2} \frac{\partial u}{\partial x}+\frac{\partial \xi}{\partial z}\left(\gamma^{2} \frac{\partial w}{\partial x}+\gamma^{3} \frac{\partial u}{\partial z}\right)\right. \\
\left.+\gamma^{4}\left(\frac{\partial \xi}{\partial z}\right)^{2} \frac{\partial w}{\partial z}\right]-\frac{\varepsilon_{1}-1}{8 \pi \gamma} N \frac{\partial \varphi_{1}}{\partial z}\left(\frac{\partial \varphi_{1}}{\partial z}-2\right) \\
\frac{\partial w}{\partial x}+\gamma^{2}\left[\frac{\partial u}{\partial x}+\frac{\partial \xi}{\partial z}\left(\frac{\partial u}{\partial x}-\frac{\partial w}{\partial z}\right)\right]=0
\end{gathered}
$$

Из (19), (20) следует, что отношение инерционных членов к силам трения по порядку величины равно $\gamma^{5} / O h$. В качестве численного примера укажем, что для касторового масла [19] $\rho=0.96 \mathrm{~g} / \mathrm{cm}^{3}, \eta=6.6 \mathrm{~g} / \mathrm{cm} \cdot \mathrm{sec}$, $\alpha=33 \mathrm{dyn} / \mathrm{cm}, O h=52.9$ при $a=0.026 \mathrm{~mm}$.

Удерживая в (18)-(20), (22) члены лидирующего порядка и возвращаясь к размерным переменным, получаeM

$$
\begin{gathered}
\frac{\partial u}{\partial x}+\frac{\partial w}{\partial z}=0, \frac{\partial p_{1}}{\partial x}=0, \eta \frac{\partial^{2} w}{\partial x^{2}}=\frac{\partial p_{1}}{\partial z}, \\
\left.\frac{\partial w}{\partial x}\right|_{x=\delta+\xi(z, t)}=0 .
\end{gathered}
$$

Из (21) следует, что в динамическом условии на свободной поверхности вклад вязких напряжений пренебрежимо мал. Ввиду этого при $N \sim 8 \pi \gamma /\left(\varepsilon_{1}-1\right)$ в размерном виде имеем при $x=\delta+\zeta(x, t)$ :

$$
\begin{aligned}
p_{1}= & -\frac{\alpha}{a^{2}}\left(\xi+a^{2} \frac{\partial^{2} \xi}{\partial z^{2}}\right)-\frac{\varepsilon_{1}-1}{8 \pi} \frac{\partial}{\partial z} \varphi_{1}(c+x, z, t) \\
& \times\left[\frac{\partial}{\partial z} \varphi_{1}(c+x, z, t)-2 E_{0}\right] .
\end{aligned}
$$

Поскольку $x=r-c$, то условия на поверхности нити (11) записываются в виде при $x=0$ :

$$
u=0, \quad w=0 .
$$

Таким образом, в случае слоя, толщина которого много меньше радиуса поперечного сечения нити, динамика жидкости описывается решением плоской задачи (14), (23)-(26). Эта задача легко преобразуется к существенно более простой постановке [8].
Из первого уравнения (23) следует

$$
u(x, z, t)=-\int_{0}^{x} \frac{\partial w}{\partial z} d x .
$$

Отметим, что на поверхности нити профиль радиальной компоненты скорости (27) удовлетворяет условию непроницаемости (26).

С учетом (27) кинематическое условие на свободной поверхности (14) записывается следующим образом:

$$
\frac{\partial \xi}{\partial t}+\frac{\partial}{\partial z} \int_{0}^{\delta+\xi(z, t)} w d x=0 .
$$

Из второго уравнения (23) следует, что распределение давления в слое не зависит от $x$. Вследствие этого функция $p_{1}(z, t)$ определяется из граничного условия на свободной поверхности (25).

Из последнего уравнения (23) с учетом (25) и граничных условий на свободной поверхности (24) и на поверхности нити (26) находим

$$
w(x, z, t)=\frac{x}{2 \eta} \frac{\partial p_{1}}{\partial z}\{x-2[\delta+\xi(x, t)]\}
$$

Используя (29) при вычислении интеграла в выражении (28) и принимая во внимание (25), получаем нелинейное уравнение

$$
\begin{gathered}
\frac{\partial \xi}{\partial t}+\frac{1}{3 \eta} \frac{\partial}{\partial z}\left\{( \delta + \xi ) ^ { 3 } \left[\frac{\alpha}{a^{2}}\left(\frac{\partial \xi}{\partial z}+a^{2} \frac{\partial^{3} \xi}{\partial z^{3}}\right)\right.\right. \\
\left.\left.-\frac{\varepsilon_{1}-1}{4 \pi} \frac{\partial^{2} \psi}{\partial z^{2}}\left(\frac{\partial \psi}{\partial z}-E_{0}\right)\right]\right\}=0, \\
\psi(z, t)=\left.\varphi_{1}(r, z, t)\right|_{r=a+\xi(z, t) .}
\end{gathered}
$$

Уравнение (30) совместно с задачей электростатики для функций $\varphi_{j}(r, z, t), j=1,2,3$ описывает изменение локальной толщины слоя с ростом времени. При численном решении уравнения (30) требуется задать начальное условие для этого уравнения - функцию $\xi(z, 0)$ и найти решение задачи электростатики, соответствующее этой начальной форме свободной поверхности.

После нахождения функций $\xi(z, t), \varphi_{j}(r, z, t), j=$ $=1,2,3$ распределение переменной части давления $p_{1}(x, z, t)$ и профили скоростей $u(x, z, t), w(x, z, t)$ вычисляются с помощью выражений (25), (27), (29).

\section{Дисперсионное соотношение}

При исследовании влияния электрического поля на развитие капиллярной неустойчивости ограничимся линейной постановкой задачи. Считая $|\xi| \ll \delta$, 
$\left|\partial \varphi_{j} / \partial z\right| \ll E_{0}, j=1,2,3$, после линеаризации уравнения (30) и задачи $(4),(5),(13)$ имеем

$$
\frac{\partial \xi}{\partial t}+\frac{\alpha \delta^{3}}{3 \eta a^{2}}\left(\frac{\partial^{2} \xi}{\partial z^{2}}+a^{2} \frac{\partial^{4} \xi}{\partial z^{4}}+\left.\frac{\varepsilon_{1}-1}{4 \pi} \frac{a^{2}}{\alpha} E_{0} \frac{\partial^{3} \varphi_{1}}{\partial z^{3}}\right|_{r=a}\right)=0
$$

$$
\frac{\partial^{2} \varphi_{j}}{\partial r^{2}}+\frac{1}{r} \frac{\partial \varphi_{j}}{\partial z}+\frac{\partial^{2} \varphi_{j}}{\partial z^{2}}=0, j=1,2,3,
$$

при $r=c$ :

$$
\varphi_{1}=\varphi_{2}, \quad \varepsilon_{1} \frac{\partial \varphi_{1}}{\partial r}=\varepsilon_{2} \frac{\partial \varphi_{2}}{\partial r}
$$

при $r=a$ :

$$
\varphi_{1}=\varphi_{3}, \quad \varepsilon_{1} \frac{\partial \varphi_{1}}{\partial r}-\frac{\partial \varphi_{3}}{\partial r}=\left(1-\varepsilon_{1}\right) E_{0} \frac{\partial \xi}{\partial z}
$$

при $\frac{r}{c} \rightarrow \infty$ :

$$
\frac{\partial \varphi_{3}}{\partial r} \rightarrow 0
$$

Предполагается, что решение $\zeta(z, t), \quad \varphi_{j}(r, z, t)$, $j=1,2,3$ задачи (31) допускает представление в виде линейных суперпозиций нормальных мод, соответственно имеющих вид

$$
\begin{gathered}
Z(k) \exp \{i[k z-\omega(k) t]\}, \\
F_{j}(r, k) \exp \{i[k z-\omega(k) t]\}, \quad j=1,2,3 .
\end{gathered}
$$

Здесь $i$ - мнимая единица, $k$ - вещественный параметр (волновое число), а функцию $\omega(k)$ требуется найти из уравнений и граничных условий (31).

Выполнив в уравнении Лапласа переход к нормальным модам, имеем

$$
\frac{d^{2} F_{j}}{d r^{2}}+\frac{1}{r} \frac{d F_{j}}{d r}-k^{2} F_{j}=0, \quad j=1,2,3
$$

Линейно независимыми решениями уравнения (32) являются модифицированные функции Бесселя $I_{0}(k r)$, $K_{0}(k r)[21]$. Учитывая условия ограниченности функций $\partial \varphi_{2} / \partial r$ при $r \rightarrow 0$ и $\partial \varphi_{3} / \partial r$ при $r \rightarrow \infty$, получаем

$$
\begin{gathered}
F_{1}=A_{1} I_{0}(k r)+B_{1} K_{0}(k r), \\
F_{2}=A_{2} I_{0}(k r), \quad F_{3}=B_{2} K_{0}(k r) .
\end{gathered}
$$

Константы $A_{1}, A_{2}, B_{1}, B_{2}$ определяются из граничных условий электростатики, записанных с использованием нормальных мод:

$$
\begin{gathered}
A_{1} I_{0}(\beta \kappa)+B_{1} K_{0}(\beta \kappa)=A_{2} I_{0}(\beta \kappa), \\
\varepsilon_{1}\left[A_{1} I_{1}(\beta \kappa)-B_{1} K_{1}(\beta \kappa)\right]=\varepsilon_{2} A_{2} I_{1}(\beta \kappa), \\
A_{1} I_{0}(\kappa)+B_{1} K_{0}(\kappa)=B_{2} K_{0}(\kappa), \\
\varepsilon_{1}\left[A_{1} I_{1}(\kappa)-B_{1} K_{1}(\kappa)\right]+B_{2} K_{1}(\kappa)=i\left(1-\varepsilon_{1}\right) E_{0} Z .
\end{gathered}
$$

Здесь и далее приняты обозначения $\kappa=k a, \beta=1-\gamma$, так что $\beta \kappa=k c$. Решение этой системы уравнений имеет вид

$$
\begin{gathered}
A_{1}=\frac{i \sigma}{d} K_{0}(\kappa)\left[\varepsilon_{1} I_{0}(\beta \kappa) K_{1}(\beta \kappa)+\varepsilon_{2} I_{1}(\beta \kappa) K_{0}(\beta \kappa)\right], \\
A_{2}=\frac{i \sigma}{d} \frac{\varepsilon_{1}}{\beta \kappa} K_{0}(\kappa), B_{1}=\frac{i \sigma}{d}\left(\varepsilon_{1}-\varepsilon_{2}\right) I_{0}(\beta \kappa) I_{1}(\beta \kappa) K_{0}(\kappa), \\
B_{2}=\frac{i \sigma}{d}\left\{\varepsilon_{1} I_{0}(\beta \kappa)\left[I_{0}(\kappa) K_{1}(\beta \kappa)+I_{1}(\beta \kappa) K_{0}(\kappa)\right]\right. \\
\left.\quad-\varepsilon_{2} I_{1}(\beta \kappa)\left[I_{0}(\beta \kappa) K_{0}(\kappa)-I_{0}(\kappa) K_{0}(\beta \kappa)\right]\right\} \\
d=\left[I_{0}(\kappa) K_{1}(\kappa)+\varepsilon_{1} I_{1} K_{0}(\kappa)\right] \\
\quad \times\left[\varepsilon_{1} I_{0}(\beta \kappa) K_{1}(\beta \kappa)+\varepsilon_{2} I_{1}(\beta \kappa) K_{0}(\beta \kappa)\right] \\
-\left(\varepsilon_{1}-1\right)\left(\varepsilon_{1}-\varepsilon_{2}\right) I_{0}(\beta \kappa) I_{1}(\beta \kappa) K_{0}(\kappa) K_{1}(\kappa), \\
\sigma=\left(1-\varepsilon_{1}\right) E_{0} Z .
\end{gathered}
$$

После перехода в (31) к нормальным модам с учетом первого выражения (33) получаем дисперсионное соотношение

$$
\omega=i \frac{\alpha \delta^{3}}{3 \eta a^{4}} \kappa^{2}\left[1-\kappa^{2}-\frac{\left(\varepsilon_{1}-1\right)^{2} \kappa}{4 \pi} N \Gamma\left(\kappa, \beta, \varepsilon_{1}, \varepsilon_{2}\right)\right],
$$

где

$$
\begin{aligned}
N= & \frac{a}{\alpha} E_{0}^{2}, \quad \Gamma=\frac{1}{d}\left\{I _ { 0 } ( \kappa ) K _ { 0 } ( \kappa ) \left[\varepsilon_{1} I_{0}(\beta \kappa) K_{1}(\beta \kappa)\right.\right. \\
& \left.\left.+\varepsilon_{2} I_{1}(\beta \kappa) K_{0}(\beta \kappa)\right]+\left(\varepsilon_{1}-\varepsilon_{2}\right) I_{0}(\beta \kappa) I_{1}(\beta \kappa) K_{0}^{2}(\kappa)\right\} .
\end{aligned}
$$

Таким образом, $\omega(k)$ является чисто мнимой функцией при всех $\kappa \neq 0$. Ввиду этого в случае $\omega_{i}=\operatorname{Im} \omega>0$ амплитуды нормальных мод начального возмущения толщины слоя экспоненциально возрастают по времени, а в случае $\omega_{i}<0-$ экспоненциально затухают. При $\omega_{i}=0$ мода с соответствующим волновым числом нейтрально устойчива.

В отсутствие электрического поля (при $N=0$ ) дисперсионное соотношение (34) с точностью до обозначений совпадает с результатом, полученным в работе [7]. В этом случае, как и в классической задаче Рэлея о неустойчивости жидкого цилиндра, неустойчивы все моды с длинами волн $\lambda>2 \pi a$.

\section{Воздействие поля на капиллярную неустойчивость}

Исследуем влияние электрического поля на развитие капиллярной неустойчивости слоя касторового масла, имеющего [22] диэлектрическую проницаемость $\varepsilon_{1}=$ 4.5. Материалом нити являются: 1) полиэтилен, у которого $\left.\varepsilon_{2}=2.3 ; 2\right)$ капрон, $\varepsilon_{2}=4.5 ; 3$ ) поливинилхлоридный пластикат изоляционный, $\varepsilon_{2}=7$ (данные приведены в [23]). В случае касторового масла при $E_{0}=15 \mathrm{kV} / \mathrm{cm}, a=0.26 \mathrm{~mm}$ имеем $N=2$. 


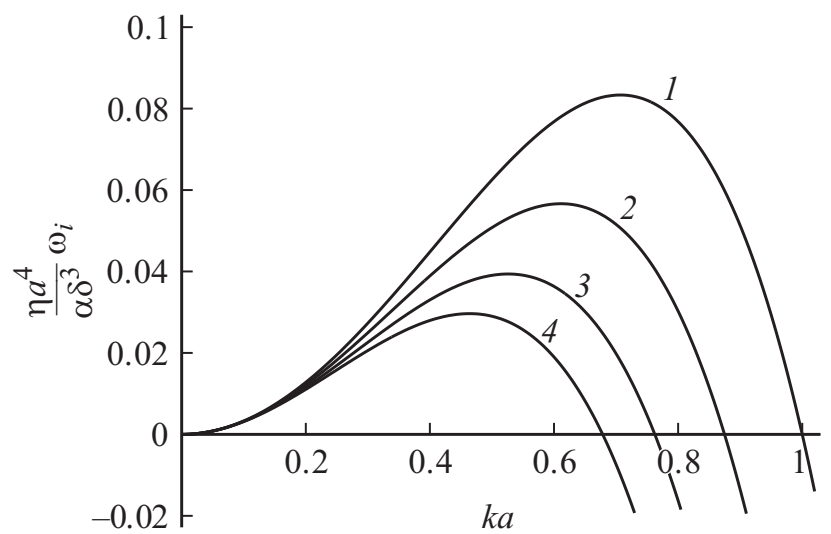

Рис. 2. Дисперсионные зависимости при $\varepsilon_{1}=4.5, \varepsilon_{2}=2.3$ и различных значениях $N: 1-0,2-0.7,3-1.4,4-2$.

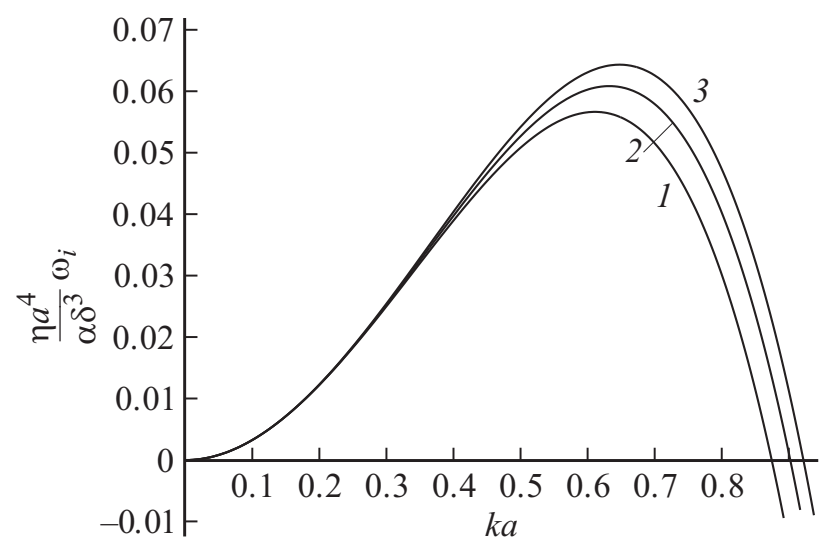

Рис. 3. Дисперсионные зависимости при $\varepsilon_{1}=4.5, N=0.7$ и различных значениях $\varepsilon_{2}: 1-2.3,2-4.5,3-7$.

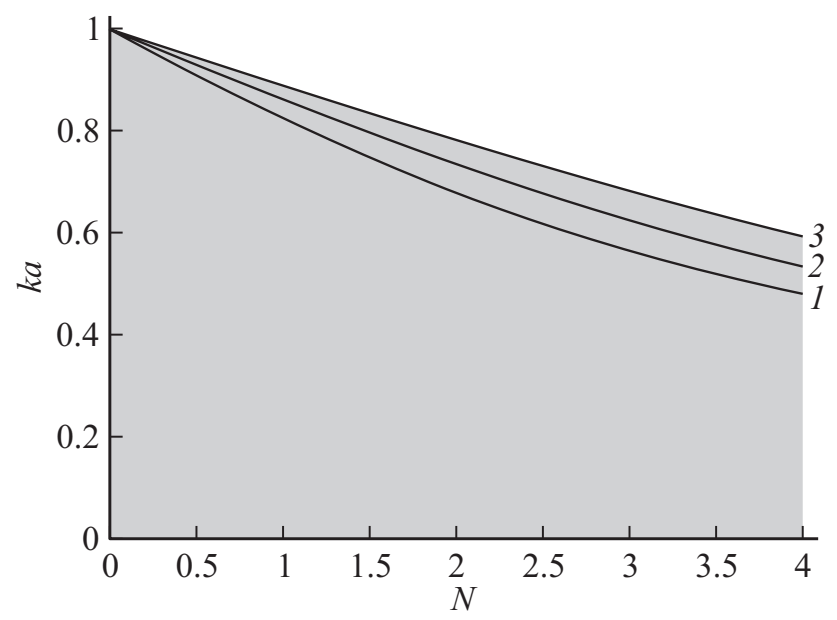

Рис. 4. Нейтральные кривые и области неустойчивости при $\varepsilon_{1}=4.5$ и различных значениях $\varepsilon_{2}: 1-2.3,2-4.5,3-7$.

В безразмерном виде дисперсионная зависимость (34) представлена на рис. 2, 3. Из графиков рис. 2 видно, что с увеличением напряженности поля волновое число $k_{n}$ нейтрально устойчивой моды уменьшается: при $N=0$ имеем $k_{n}=a^{-1}$, тогда как $k_{n}<a^{-1}$ при $N>0$. В результате при фиксированных $\varepsilon_{1}, \varepsilon_{2}$ с ростом напряженности поля диапазон неустойчивых мод сужается.

Взаимное расположение дисперсионных кривых на рис. 3 показывает, что увеличение диэлектрической проницаемости материала нити $\varepsilon_{2}$ при фиксированных $N$ и $\varepsilon_{1}$ вызывает дестабилизацию, поскольку при этом диапазон неустойчивых мод расширяется. Количественно этот эффект показан на рис. 4, где области неустойчивости, построенные в плоскости параметров $N, k a$, выделены темным цветом. Границами областей неустойчивости являются оси координат и соответствующие выбранным значениям $\varepsilon_{2}$ кривые нейтральной устойчивости, вдоль которых $\omega_{i}=0$.

При исследовании капиллярного распада осесимметричного жидкого слоя особый интерес представляет волновое число $k_{m}$ наиболее быстро растущей моды, реализующее при заданных $\varepsilon_{1}, \varepsilon_{2}, N$ максимум дисперсионной кривой. В линейной постановке ввиду доминирующего влияния этой моды на развитие неустойчивости ее пространственный масштаб $\lambda_{m}=2 \pi / k_{m}$ является характерным расстоянием между периодически располагающимися вдоль нити капельками, формирующимися на финальной стадии развития неустойчивости. В настоящей работе, как и при обработке экспериментальных данных в [6], образование сателлитов не учитывается.

При $N=0$ из дисперсионного соотношения (34) легко находятся $k_{m}^{0}=(\sqrt{2} a)^{-1}, \lambda_{m}^{0}=2 \sqrt{2} \pi a$, что совпадает с величиной, приведенной в [6] для предельного $(\delta \rightarrow 0)$ случая тонкого слоя. Скорость роста этой моды $s_{0}=\omega_{i}\left(k_{m}^{0}\right)$ определяется выражением $s_{0}=\alpha \gamma^{3} /(12 \eta a)$.

Из построенных на рис. 5 графиков следует, что при фиксированных $\varepsilon_{1}, \varepsilon_{2}$ с ростом напряженности поля расстояние между капельками увеличивается. На этом рисунке кривая 2 соответствует случаю $\varepsilon_{1}=\varepsilon_{2}$, когда при переходе границы между нитью и жидкостью нормальная компонента возмущения поля не испытывает

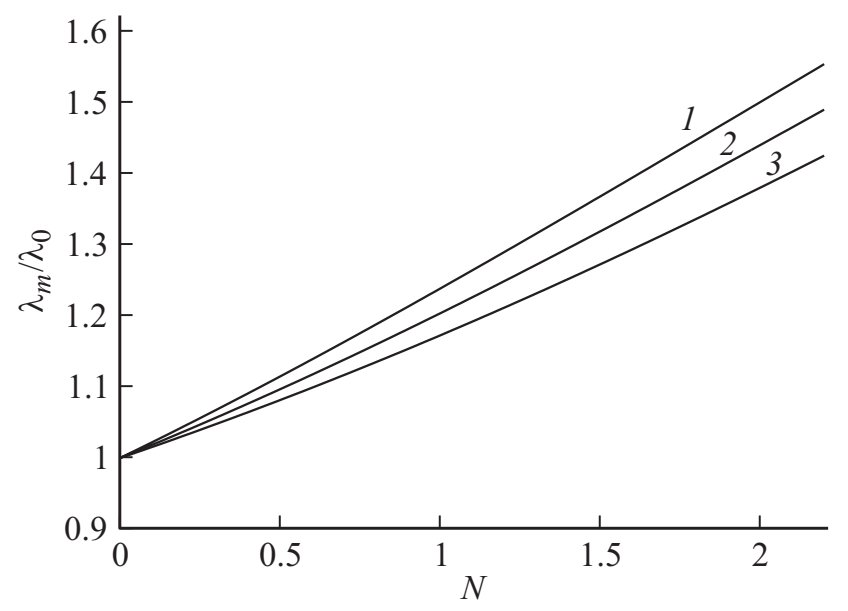

Рис. 5. Зависимость от $N$ нормированной длины волны наиболее быстро растущей моды при $\varepsilon_{1}=4.5$ и различных значениях $\varepsilon_{2}: 1-2.3,2-4.5,3-7$. 


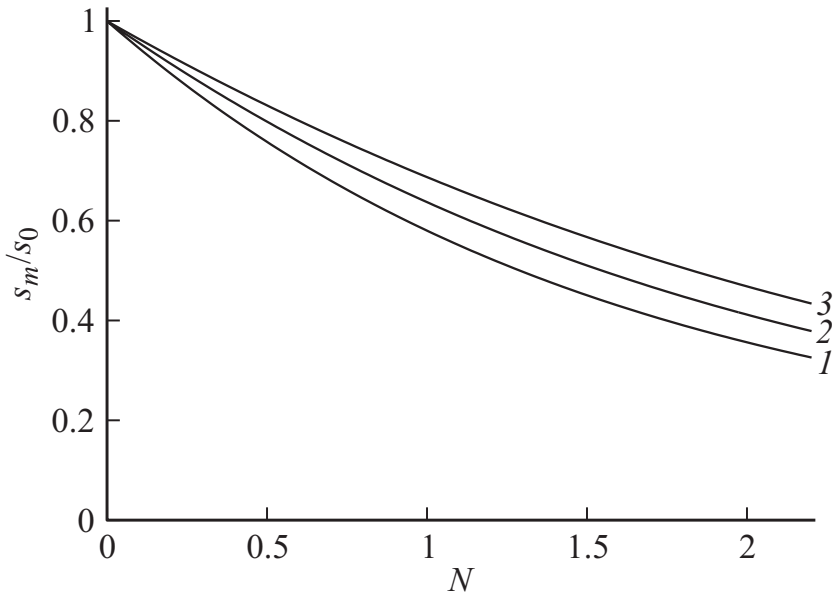

Рис. 6. Зависимость от $N$ нормированной скорости роста наиболее быстро растущей моды при $\varepsilon_{1}=4.5$ и различных значениях $\varepsilon_{2}: 1-2.3,2-4.5,3-7$.

скачка. Из графиков следует, что при фиксированной напряженности поля расстояние между соседними капельками в случае $\varepsilon_{2}<\varepsilon_{1}$ оказывается больше, чем в случае $\varepsilon_{2}>\varepsilon_{1}$.

На рис. 6 представлены графики зависимости от $N$ нормированной скорости роста $s_{m} / s_{0}$ наиболее быстро растущей моды, построенные при фиксированном $\varepsilon_{1}$ и различных $\varepsilon_{2}$. Графики показывают, что величина $s_{m} / s_{0}$ уменьшается с ростом напряженности поля тем значительное, чем меньше диэлектрическая проницаемость материала нити.

Графики на рис. 2-6 построены при $\beta=0.93$. При малых $\gamma$ влияние параметра $\beta$ на дисперсионные зависимости незначительно.

\section{Заключение}

Применительно к случаю малых чисел Бонда изучено влияние однородного продольного электрического поля $\mathbf{E}_{0}$ на капиллярную неустойчивость осесимметричного слоя вязкой диэлектрической жидкости, не содержащей свободных электрических зарядов. Слой покрывает прямолинейную диэлектрическую нить цилиндрической формы и окружен снаружи газом, находящимся при постоянном давлении. Диэлектрические проницаемости жидкости $\varepsilon_{1}$ и материала нити $\varepsilon_{2}$ в общем случае различны. Средняя толщина слоя много меньше радиуса поперечного сечения нити.

В предположении о балансе сил трения и продольного градиента давления (что реализуется при гидродинамической смазке [9]) проведены оценки порядков величин в уравнениях и граничных условиях гидродинамики, учитывающих поверхностные пондеромоторные силы.

На базе постановки задачи, учитывающей только члены лидирующего порядка, выведено нелинейное параболическое уравнение, описывающее эволюцию с ростом времени возмущения постоянной в начальный момент толщины слоя. Распределения поля в нити, в слое и в окружающем слой газе описываются уравнениями и граничными условиями электростатики.

В рамках линеаризованного эволюционного уравнения и задачи электростатики с линеаризованными граничными условиями на свободной поверхности слоя получено дисперсионное соотношение и проведен его анализ.

Показано, что электрическое поле стабилизирует некоторый диапазон неустойчивых в отсутствие поля нормальных мод с пространственными масштабами, превышающими периметр внешней границы кольца, представляющего поперечное сечение исходного слоя постоянной толщины. При фиксированных $\varepsilon_{1}, \varepsilon_{2}$ с ростом $E_{0}$ диапазон стабилизируемых полем мод увеличивается. Если же $E_{0}$ и $\varepsilon_{1}$ фиксированы, то увеличение диэлектрической проницаемости материала нити $\varepsilon_{2}$ оказывает дестабилизирующее воздействие.

При фиксированном $\varepsilon_{1}$ и различных значениях $\varepsilon_{2}$ в плоскости параметров: электрокапиллярное число безразмерное волновое число построены кривые нейтральной устойчивости, разделяющие области неустойчивости, соответствующие различным $\varepsilon_{2}$.

Установлено, что при фиксированных $\varepsilon_{1}, \varepsilon_{2}$ с ростом $E_{0}$ увеличивается пространственный масштаб наиболее быстро растущей моды, что приводит к увеличению расстояния между соседними капельками, формирующимися на финальной стадии развития капиллярной неустойчивости слоя. Если же $E_{0}$ и $\varepsilon_{1}$ фиксированы, а $\varepsilon_{2}$ изменяется, то в случае $\varepsilon_{2}<\varepsilon_{1}$ расстояние между капельками оказывается больше, чем в случае $\varepsilon_{2}>\varepsilon_{1}$. Показано также, что при фиксированной диэлектрической проницаемости жидкости с увеличением $E_{0}$ скорость роста наиболее быстро растущей моды уменьшается тем значительнее, чем меньше величина диэлектрической проницаемости материала нити.

Работа выполнена при поддержке Российского фонда фундаментальных исследований (проект № 14-0100056).

\section{Список литературы}

[1] Бойс Ч. Мыльные пузыри. Петроградъ: Научное книгоиздательство, 1919. 192 с.; Boys C.V. Soap bubbles and the forces which mould them. New Delhi: Vigyan Prasar, 2002. $96 \mathrm{p}$.

[2] Стретт Джс.В. (Лорд Рэлей). Теория звука. Т. 2. М.: ГИТТЛ, 1955. $475 \mathrm{c}$.

[3] Дразин Ф. Введение в теорию гидродинамической устойчивости. М.: Физматлит, 2005. $287 \mathrm{c}$.

[4] Бондаренко В.С. // ЖФХ. 1961. Т. 35. № 12. С. 2775-2777.

[5] Quéré D. // Annu. Rev. Fluid Mech. 1999. Vol. 31. P. 347384.

[6] Goren S.L. // J. Fluid Mech. 1962. Vol. 12. N 2. P. 309-319.

[7] Yarin A.L., Oron A., Rosenau Ph. // Phys. Fluid. A. 1993. Vol. 5. N 1. P. 91-98. 
[8] Oron A., Davis S.H., Bankoff S.G. // Rev. Mod. Phys. 1997. Vol. 69. N 3. P. 931-980.

[9] Шлихтинг Г. Теория пограничного слоя. М.: Наука, 1969. $742 \mathrm{c}$.

[10] Saville D.A. // Phys. Fluid. 1970. Vol. 13. N 12. P. 2987-2994.

[11] Savettaseranee K., Papageorgiou D.T., Petropoulos P.G., Tilley B.S. // Phys. Fluid. 2003. Vol. 15. N 3. P. 641-652.

[12] Коровин В.М. // ЖТФ. 2011. Т. 81. Вып. 10. С. 12-19.

[13] Сивухин Д.В. Электричество. М.: Наука, 1983. 688 с.

[14] Saville D.A. // Annu. Rev. Fluid. Mech. 1997. Vol. 29. P. 2764.

[15] Tilley B.S., Petropoulos P.G., Papageorgiou D.T. // Phys. Fluid. 2001. Vol. 13. N 12. P. 3547-3563.

[16] Papageorgiou D.T., Vanden-Broeck J.-M. // J. Fluid. Mech. 2004. Vol. 508. P. 71-88.

[17] Зубарев Н.М. // Письма в ЖЭТФ. 2009. Т. 89. Вып. 6. C. $317-321$

[18] Ландау Л.Д., Лифиии, Е.М. Электродинамика сплошных сред. М.: Наука, 1992. 664 с.

[19] Goren S.L. // J. Colloid Sci. 1964. Vol. 19. P. 81-86.

[20] Седов Л.И. Механика сплошной среды. Т. 1. М.: Наука, 1983. $528 \mathrm{c}$.

[21] Справочник по специальным функциям / Под ред. М. Абрамовица, И. Стиган. М.: Мир, 1979. 830 с.

[22] Таблицы физических величин / Под ред. И.К. Кикоина. М.: Атомиздат, 1976. $1008 \mathrm{c}$.

[23] Физические величины / Под ред. И.С. Григорьева, Е.3. Мейлихова. М.: Энергоатомиздат, 1991. 1232 с. 